\title{
REMARKS ON A THEOREM OF M. ALTMAN
}

\section{REICHBACH}

Introduction. A mapping ${ }^{1} f: X \rightarrow X$ of a Banach space $X$ into itself is said [1] to be locally an $\epsilon$-mapping in the narrow sense, if for every $x \in X$ there exist two positive numbers $\eta_{x}$ and $\epsilon_{x}$ such that the condition

$$
x^{\prime}, x^{\prime \prime} \in S\left(x, \epsilon_{x}\right) \text { and }\left\|f\left(x^{\prime}\right)-f\left(x^{\prime \prime}\right)\right\|<\eta_{x}
$$

implies

$$
\left\|x^{\prime}-x^{\prime \prime}\right\|<\epsilon_{x},
$$

where $S\left(x, \epsilon_{x}\right)$ denotes the spherical region (in $X$ ) with centre $x$ and radius $\epsilon_{x}$.

In [1] M. Altman proved that: If the mapping $f(x)=x-F(x)$, $x \in X$, where $F(x)$ is a completely continuous transformation of the Banach space $X$ with range in $X$, is locally an $\epsilon$-mapping in the narrow sense, then the image $f(X)$ of $X$ is an open subset of the space $X$.

Using this theorem he obtained in narticular some conditions under which a linear mapping $f: X \rightarrow X$ of $X$ into $X$ is a mapping onto $X{ }^{2}$

In this paper, the definition of locally an $\epsilon$-mapping in the narrow sense is slightly modified and some theorems on mappings onto are proved for the so-called polynomial mappings. ${ }^{3}$ It turns out that for these mappings the assumption that the mapping is locally an $\epsilon$ mapping in the narrow sense in every point $x \in X$ (made in Altman's theorems referred to above) may be replaced by a much weaker assumption.

I. Definition 1. A mapping $f: X \rightarrow Y$ of a metric space $X$ with metric $\rho_{1}$ into a metric space $Y$ with metric $\rho_{2}$ will be called an $\epsilon$ mapping in the narrow sense at the point $y \in f(X)$, if there exists a point $x \in f^{-1}(y)$ and two positive numbers $\eta_{x}$ and $\epsilon_{x}$ such that

$$
x^{\prime}, x^{\prime \prime} \in S\left(x, \epsilon_{x}\right) \text { and } \rho_{2}\left[f\left(x^{\prime}\right), f\left(x^{\prime \prime}\right)\right]<\eta_{x}
$$

implies

$$
\rho_{1}\left(x^{\prime}, x^{\prime \prime}\right)<\epsilon_{x},
$$

Received by the editors May 5, 1960.

1 By "mapping" we understand always a continuous mapping.

2 See $[1$, p. 1039, Corollary 1].

${ }^{3}$ See $[5$, p. 157]. 
where $S\left(x, \epsilon_{x}\right)$ denotes the spherical region (in $X$ ) with centre $x$ and radius $\epsilon_{x}$.

Note that if $f: X \rightarrow X$ is locally an $\epsilon$-mapping in the narrow sense, then it is also an $\epsilon$-mapping in the narrow sense at every point $y \in f(X)$-but, as can be easily seen, not conversely.

Definition 2. A mapping $f: X \rightarrow Y$ of a metric space $X$ into a metric space $Y$ is called open at the point $y \in f(X)$ if there exists a spherical region $S(y, r)$, (in $Y$ ), with centre $y$ and radius $r$ such that $S(y, r)$ $C f(X)$.

THEOREM 1. If $F: X \rightarrow X$ is a completely continuous operator of a Banach space $X$ into itself and $f(x)=x-F(x)$ is an e-mapping in the narrow sense at the point $y \in f(X)$, then $f: X \rightarrow X$ is open at the point $y \in f(X)$.

The proof of this theorem is identical with that of Theorem 1 in [1].

Definition 3. A mapping $f: X \rightarrow Y$ of a metric space $X$ into a metric space $Y$ is called a polynomial mapping if the condition:

$\left\{x_{n}\right\}_{n=1,2}, \ldots$ does not contain a Cauchy (fundamental) subsequence, implies that

$$
\left\{f\left(x_{n}\right)\right\}_{n=1} 2, \ldots \text { also does not contain a Cauchy subsequence. }
$$

We give now some examples of polynomial mappings:

1. A mapping $f: X \rightarrow Y$ is called an $\epsilon$-mapping in the narrow sense ${ }^{4}$ if there exist two positive numbers $\eta$ and $\epsilon$ such that the condition $\rho_{2}\left[f\left(x^{\prime}\right), f\left(x^{\prime \prime}\right)\right]<\eta, x^{\prime}, x^{\prime \prime} \in X$ implies $\rho_{1}\left(x^{\prime}, x^{\prime \prime}\right)<\epsilon$, where $\rho_{1}$ and $\rho_{2}$ denote the metric in $X$ and $Y$ respectively. We shall show that an $\epsilon$-mapping $f: X \rightarrow Y$ in the narrow sense of a finite dimensional Banach space $X$ into a metric space $Y$ is a polynomial mapping.

Indeed, if $\left\{f\left(x_{n}\right)\right\}_{n=1,2}, \ldots$ contains a Cauchy subsequence $\left\{f\left(x_{n}^{\prime}\right)\right\}_{n=1,2}, \ldots$, then for any $\eta$ there is $\rho_{2}\left[f\left(x_{n}^{\prime}\right), f\left(x_{m}^{\prime}\right)\right]<\eta$ for $n, m$ sufficiently large. Hence $\rho_{1}\left(x_{n}^{\prime}, x_{m}^{\prime}\right)=\left\|x_{n}^{\prime}-x_{m}^{\prime}\right\|<\epsilon$ and therefore $\left\{x_{n}^{\prime}\right\}_{n=1,2}, \ldots$ is a bounded sequence in $X$. Thus, $X$ being a finite dimensional Banach space, the sequence $\left\{x_{n}^{\prime}\right\}$ is compact (conditionally) and therefore it contains a convergent subsequence $\left\{x_{n}^{\prime \prime}\right\}_{n=1,2}, \ldots$ which is a Cauchy subsequence of $\left\{x_{n}\right\}_{n=1,2}, \ldots$

2. Every mapping $f: X \rightarrow Y$ of a finite dimensional Banach space $X$ into a finite dimensional Banach space $Y$, such that if $\left\|x_{n}\right\| \rightarrow \infty$ then also $\left\|f\left(x_{n}\right)\right\| \rightarrow \infty$, is a polynomial mapping. ${ }^{5}$

3. If there exists a constant $k>0$ such that

4 See [2].

See $[6$, p. $1398,(c)]$. 


$$
\rho_{2}\left[f\left(x^{\prime}\right), f\left(x^{\prime \prime}\right)\right] \geqq k \rho_{1}\left(x^{\prime}, x^{\prime \prime}\right)
$$

then $f: X \rightarrow Y$ is a polynomial mapping ( $\rho_{1}$ and $\rho_{2}$ denoting the metrics in $X$ and $Y$ respectively).

Theorem 2. If $f: X \rightarrow Y$ is a polynomial mapping of a complete metric space $X$ into a connected metric space $Y$ which is open at every point $y \in f(X)-J$, where $J \neq f(X)$ and $J \subset f(X)$ is a set which does not disconnect the space $Y$, then $f(X)=Y .^{6}$

Proof. Since $J \neq f(X)$ and $f: X \rightarrow Y$ is open at every point $\bar{y}$ of $f(X)-J$, there exists a spherical region $S(\bar{y}, r)$ (in $Y$ ) which is contained in $f(X)$. Denote by $U$ the union of all sets, open in $Y$, which are contained in $f(X)$. Evidently $\operatorname{Fr}(U) \subset \operatorname{Fr}[f(X)] .{ }^{7}$ Now suppose that there exists a point $y_{0} \in Y-f(X)$ and let $y_{1}$ be any point belonging to $U$. Since the set $J$ does not disconnect $Y$, there exists in $Y-J$ a connected set $C$ containing $y_{0}$ and $y_{1}$. But the set $\operatorname{Fr}(U)$ disconnects the space $Y$ between $y_{0}$ and $y_{1} ;{ }^{8}$ therefore, there exists a point $y_{2} \in[\operatorname{Fr}(U)-J] \cap C \subset \operatorname{Fr}[f(X)]-J$. By Theorem 1 of $[5]^{9} f(X)$ is closed in $Y$. Hence $y_{2} \in f(X)$. By assumption, $f: X \rightarrow Y$ is open at every point $y \in f(X)-J$ in contradiction with the fact that

$$
y_{2} \in \operatorname{Fr}[f(X)]-J \subset f(X)-J,
$$

because $f: X \rightarrow Y$ is evidently not open at every point belonging to Fr $[f(X)]$. Thus the assumption that there exists a point $y_{0} \in Y-f(X)$ leads to a contradiction. From Theorems 1 and 2 we obtain the following

Theorem 3. If $F: X \rightarrow Y$ is a completely continuous operator of a Banach space $X$ into itself and $f(x)=x-F(x)$ is a polynomial mapping which is also an e-mapping in the narrow sense at every point

$$
y \in f(X)-J,
$$

where $J \neq f(X)$ and $J \subset f(X)$ is a set which does not disconnect $X$, then $f(X)=X$.

Indeed, by Theorem $1, f: X \rightarrow X$ is open at every point $y \in f(X)-J$ and hence by Theorem 2 used for $X=Y$, there is $f(X)=X$.

REMARK 1. The function $f(x)=\arctan x(F(x)=x-\arctan x)$ de[6].

- Some generalizations of this theorem to general topological spaces will be given in

${ }^{7} \operatorname{Fr}(A)$ denotes the boundary of $A$ in $Y$, i.e., $\operatorname{Fr}(A)=\bar{A} \cap \overline{Y-A}$.

${ }^{8}$ See [3, p. 247]; also [4, p. 80].

See $[5$, p. 158]. 
fined on the real line $X$ is an example of a function which is locally an $\epsilon$-mapping in the narrow sense, but which is not a polynomial mapping. In this case we have $f(X) \neq X$.

REMARK 2. From Theorem 3 it follows that if $F$ satisfies the assumptions of Theorem 3 , then there exists a point $x$ such that $F(x)=x$ (i.e., $F: X \rightarrow X$ has a fixed point).

\section{REFERENCES}

1. M. Altman, On a theorem of K. Borsuk, Bull. Acad. Polon. Sci. Cl. III vol. 5 (1957) pp. 1037-1040.

2. K. Borsuk, Ueber stetige Abbildungen der Euklidischen Raeume, Fund. Math. vol. 21 (1933) pp. 236-243.

3. F. Hausdorff, Grundzuege der Mengenlehre, New York, Chelsea, 1949.

4. C. Kuratowski, Topologie. II, Warszawa, Monografie Matematyczne, 1952.

5. M. Reichbach, Generalizations of the fundamental theorem of algebra, Bull. Res. Council Israel vol. 7 (1958) pp. 155-164.

6. - Some theorems on mappings onto, Pacific J. Math. vol. 10 (1960) pp. 1397-1407.

Technion-Israel Institute of Technology, Haifa, Israel

\section{A CHAINABLE CONTINUUM NO TWO OF WHOSE NONDEGENERATE SUBCONTINUA ARE HOMEOMORPHIC}

\section{JAMES J. ANDREWS}

R. D. Anderson and Gustave Choquet [1] gave an example of a plane continuum no two of whose nondegenerate subcontinua are homeomorphic. The object of this note is to point out that there is a chainable continuum having this property. The only change we make in the construction given in [1] is to replace the $n$-ods used by R. D. Anderson and Gustave Choquet by chainable continua $C_{n-2}$.

A subcontinuum $Y$ of a continuum $X$ is a separating continuum of $X$ if $X-Y$ is not connected and $\mathrm{Cl}(X-Y)=X$. A subcontinuum $Y$ of a continuum $X$ is a strong separating continuum if:

(1) $Y$ is a separating continuum of $X$,

(2) $X-Y$ has two components, $X_{1}$ and $X_{2}$,

(3) there are points $y_{1}, y_{2} \in Y$ such that $y_{i} \notin \mathrm{Cl}\left(X_{i}\right)$.

Let $V=\{(x, y) / y=|x|$ and $-1<x<1\}$. Let $C_{n}$ be formed from $n$ copies of $V$ and $n+1$ "lines" so that each $V$ is a strong separating continuum of $C_{n}$ as in Figure 1 (for $n=2$ ).

Received by the editors May 10, 1960. 